strong evidence that mild steel welding fumes, in addition to stainless steel, induce chronic inflammation and are immunosuppressive, and this was confirmed in molecular epidemiology studies of workers. We continued studies using metabolomic approaches in a repeated measures design and found welding fume exposure-related changes in blood in pathways related to disturbances in unsaturated fat metabolism, as in the signaling lipids Sphingosine 1-phosphate (S1P) and sphingasine 1-phosphate (SA1P). Global metabolomic profiling also revealed several metabolic changes after welding fume exposure, mainly involved in the lipid pathway [glucocorticoid class (cortisol, corticosterone, and cortisone), acylcarnitine class, and DiHOME species (9,10-DiHOME and 12,13-DiHOME)], amino acid utilization (isoleucine, proline and phenylalanine), and S-(3-hydroxypropyl) mercapturic acid (3-HPMA): compounds are all associated with inflammation.

Conclusion There is strong mechanistic evidence in humans for inflammatory and metabolic changes that promote carcinogenicity of welding fumes in humans.

\section{S-495 INTRODUCTION TO THE KEY CHARACTERISTICS APPROACH TO INTERPRETING MECHANISTIC DATA}

${ }^{1}$ Martyn Smith. ${ }^{1}$ University of California, Berkeley School of Public Health, United States

\subsection{6/OEM-2021-EPI.449}

The key characteristics (KCs) of human carcinogens were recently introduced as the basis of a uniform approach for searching, organizing, and evaluating mechanistic evidence to support cancer hazard identification (PMID:30521319). The KCs comprise the properties of known human carcinogens, including their ability to, be genotoxic; be immunosuppressive; or modulate receptor-mediated effects (PMID: 26600562). Established human carcinogens commonly exhibit one or more of these characteristics, and therefore, data on these characteristics can provide independent evidence of carcinogenicity when human data are lacking (PMID: 29562322). Such data can also help in interpreting the relevance and importance of findings of cancer in animals and in humans. In its 2017 report on 'Using 21st Century Science to Improve Risk-Related Evaluations', the NRC opined that the KCs approach 'avoids a narrow focus on specific pathways and hypotheses and provides for a broad, holistic consideration of the mechanistic evidence.' They further suggested that key characteristics be developed for other endpoints, such as endocrine disruption and reproductive toxicity. These have recently been published (PMID: 31719706; 31322437; 31199676) and KCs for hepato-, immuno-, neuro- and cardiovascular toxicants are in the final stages of development. We have also recently published the findings of two expert committees who described approaches to studying carcinogenicity of chemical mixtures using the KCs (PMID: 33784186) and identified biomarkers that can be used to measure the KCs of carcinogens in humans, animals and cell culture (PMID: 32152214). A uniform approach to applying these biomarkers in occupational studies of different epidemiologic design needs to be developed so that the most relevant biomarkers of each KC are measured in exposed human populations, thereby improving hazard identification and risk assessment. FROM SIX CONTINENTS

${ }^{1}$ Annesi-Maesano. 'INSERM, France

\subsection{6/OEM-2021-EPI.450}

Objectives Farming is filled with respiratory hazards: pesticide vapors, dusty fields, dangerous hydrogen sulfide accumulations in manure pits and pump sumps, nitrogen dioxide in conventional silos, and many others. Despite the recognition of respiratory hazards, this problem has not been fully investigated at the world level after harmonization of data. We used data from the AGRICOH consortium, a collective of prospective cohorts of agricultural workers, to assess respiratory disease prevalence among adults in 18 cohorts representing over 200,000 (118,520 men, 92,712 women) farmers, farmworkers, and their spouses from six continents.

Methods Cohorts collected data between 1992-2016 and ranged in size from 200 to $>128,000$ individuals; $44 \%$ of participants were female. Farming practices varied from subsistence farming to large scale industrial agriculture. All cohorts provided respiratory outcome information for their cohort based on their study definitions. The majority of outcomes were based on self-report using standard respiratory questionnaires; the greatest variability in assessment methods was associated with chronic obstructive pulmonary disease (COPD).

Results The median prevalence was $18.6 \%$ for cough, $13.3 \%$ for phlegm, and $15.0 \%$ for wheeze and was higher in men than in women, with the greatest difference for phlegm $(17 \%$ vs. $10 \%)$. For asthma, the median prevalence was $7.2 \%$ and was higher in women $(7.8 \%$ vs. $6.5 \%)$. The relative proportion of allergic asthma varied among cohorts. In two of eight cohorts for women and two of seven cohorts for men, allergic asthma was more common than non-allergic asthma. The median prevalence of COPD was $4.5 \%$. Men suffered more than women from COPD (5.5\% vs. $4.0 \%)$.

Conclusion These findings indicate that respiratory outcomes are common among farmers around the world despite the differences in agricultural production. Both allergic and non-allergic asthma exist among farmers.

\section{S-497 COMPARISON OF REPORTED RELATIVE RISKS FOR HEALTH CARE, TRANSPORT, AND FOOD PROCESSING WORKERS}

${ }^{1} S$ Rhodes, M Gittins, K Stocking, M Van Tongeren, N Pearce. 'The University of Manchester, United Kingdom

\subsection{6/OEM-2021-EPI.451}

Background Risk of SARS-CV-2 infection has been strongly linked to occupation, with specific occupational sectors such as health care, food production, and transport, particularly affected. To better understand the potential risks by occupational sector we investigated the reported risks of COVID-19 infection and mortality for employees in the three sectors.

Methods We performed a rapid review of observational studies reporting COVID-19 risk for employees in health care, food manufacturing, and transport sectors. All studies published in the peer-review and pre-print literature between March 2020 and June 2021 were considered. The primary outcome measure was COVID-19 infection, with COVID-19 related mortality and hospitalisation considered as secondary measures. We 